Proceedings of the XXIII Conference on Applied Crystallography, Krynica Zdrój, Poland, September 20-24, 2015

\title{
Microstructure Characterization of Noble Metal-Silica Nanocomposites
}

\author{
M. ZienKiEWICZ-STRZAŁKA ${ }^{a, *}$ AND S. PikUS ${ }^{b}$ \\ ${ }^{a}$ Department of Physicochemistry of Solid Surface, Faculty of Chemistry, Maria Curie-Skłodowska University, \\ Lublin, Poland \\ ${ }^{b}$ Department of Crystallography, Faculty of Chemistry, Maria Curie-Skłodowska University, Lublin, Poland
}

The presence of highly dispersed metal particles on solid supports with well-defined microstructure is important in the field of functional materials, active catalysts as well as bionanomaterials for medical applications. Noble metal nanostructures, in particular silver, palladium, and platinum nanoparticles were formed from ammine complexes $\left(\left[\mathrm{Pt}\left(\mathrm{NH}_{3}\right)_{4}\right] \mathrm{Cl}_{2},\left[\mathrm{Ag}\left(\mathrm{NH}_{3}\right)_{2}\right] \mathrm{OH}\right.$, and $\left.\left[\mathrm{Pd}\left(\mathrm{NH}_{3}\right)_{4}\right] \mathrm{Cl}_{2}\right)$ and supported on high ordered mesoporous silica (SBA-15) and aluminosilica matrix. In this work, the distribution, composition and crystal structure of supported noble metal nanoparticles were determined and characterized. Finally the stability of incorporated nanostructures was confirmed. The microstructures of the obtained samples were analyzed by high resolution transmission electron microscopy. Obtained results indicated that developed procedures of synthesis and modification of mesoporous ordered silica or their derivate by proposed nanostructures are effective and allow to obtain new nanocomposites and nanocatalysts in repeatable and controlled way.

DOI: 10.12693/APhysPolA.130.972

PACS/topics: 61.46.Df

\section{Introduction}

Nanotechnology and materials with new and unique structural properties are an essential part of modern science and inscribed on a permanent basis in actual trends and directions of research [1]. For many years, nanotechnology has become an interdisciplinary field of science linking material science, human health, physics and environmental issues due to their significant physical, chemical, and biological properties [2-5]. Although nanoparticles of noble metals were known and appreciated for centuries, the features and phenomena responsible for these uncommon properties are still being investigated and recognized [6, 7]. Synthesis and applications of composites containing metallic nanoparticles fit perfectly into this trend. In the field of nanoscale materials, noble metal nanoparticles have played an important role in the enhancement of materials functionality [8-10]. A wellknown is the fact that unique properties of nanoparticles are related with their stability. One method for stabilization the metallic nanoparticles is their deposition on a solid support [11]. The specific properties of solid material may determine the final properties of the nanoparticles. This issue is interesting especially when solid support exhibit well-defined surface properties such as high specific surface area and ordered porous structure. In this case, the size of pores determine the diameter and shape of particles and solid surface is responsible for stabilization of nanostructures. Latest achievements in designing and controlling the size and shape of nanoparticles have opened the possibility to clarify their geometry which

\footnotetext{
*corresponding author; e-mail: gosiazienkiewicz@wp.pl
}

is directly related to chemical activity. Microstructural characterization of noble metal nanoparticles is important for understanding their unique structures and properties $[12,13]$.

In this work we applied high ordered mesoporous silica and new type of zeolite/silica composites supports as a groundwork of noble metal nanoparticles from unusual noble metal precursors. At this point we focused on the characterization of noble metals nanoparticles deposited on solid surface by TEM imaging. It is significant to investigate the microstructure of noble metal nanoparticles prepared from ammine complexes because the properties of nanoparticles depend strongly on the details of its microstructure, chemical composition, particle size, shape, and agglomeration. In consequence, microstructural characterization gives the possibility for obtaining nanoparticles with desired physical and chemical properties. Detailed information about systems of noble metal/silica composites, especially in the term of support-metal nanoparticles interactions and examples of applications were presented in our other work [14-16].

\section{Experimental}

Mesoporous ordered silica SBA-15 was prepared by sol-gel procedure and described in detail in our previous work [17]. Mesoporous aluminosilicate composite was prepared by similar way in the presence of zeolite (in the form of $\mathrm{NH}_{4} \mathrm{Y}$ ) during synthesis of mesoporous ordered silica. The aim of zeolites addition was obtaining a new type of materials with great amount of micropores. Noble metal nanoparticles were introduced by impregnation of solid support by noble metal ions and their reduction to metallic state. Transmission electron microscopy with energy-dispersive X-ray analysis (TEM/EDX) was 
carried out on a high resolution scanning transmission electron microscope Titan G2 60-300 (FEI). High resolution TEM (HRTEM) measurements were performed at accelerating voltages $300 \mathrm{kV}$. The samples were prepared by dropping solution (solid sample in ethanol) on the copper grid covered with amorphous carbon and dried in air. The composites were analyzed by X-ray fluorescence spectroscopy (XRF) using the Axios (PANalytical, 2012) fluorescence spectrometer and X-ray diffraction (XRD) performed by Empyrean difractometer (PANalytical, 2013) with $\mathrm{Cu} K_{\alpha}$ radiation $(\lambda=1.5418 \AA)$ in the wide range of $2 \theta$.

\section{Results and discussion}

Application of ammine complexes as a precursors of nanoparticles is a chance to use one type of precursor for various forms of nanometals. The ammine complexes adsorption procedure of noble metals and their reduction in hydrogen atmosphere generate very small nanoparticles. Platinum nanoparticles with very small size (approximately $2 \mathrm{~nm}$ ) are homogeneously distributed on the silica surface (Fig. 1).

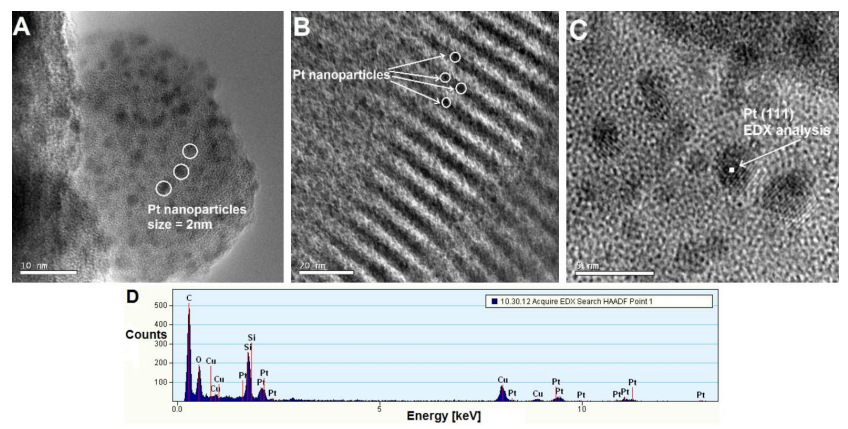

Fig. 1. (A,B) TEM images of the prepared platinum nanoparticles supported on SBA-15, (C) HRTEM images of platinum nanoparticles, (D) EDX analysis from single platinum nanoparticles.

Nanoparticles are visible on TEM images as small dark objects and represent single platinum nanoparticles with well-defined crystal structure (Fig. 1C). HRTEM images depicted lattice planes with interplanar spacing $d_{\mathrm{Pt}(111)}=0.26 \mathrm{~nm}$. The chemical composition was confirmed by TEM/EDX analysis (Fig. 1D). The presence of small platinum nanoparticles was also confirmed by X-ray diffraction measurements. Diffraction peaks showed low intensity at relatively large full width at half maximum. The half width values were determined as 4.48, 4.23, and $5.342 \theta$ degrees for $\mathrm{Pt}(111), \operatorname{Pt}(200)$ and $\mathrm{Pt}(220)$ which indicate that the size of the crystallites is at $1.9,2.0$, and $1.8 \mathrm{~nm}$, respectively.

Silver nanoparticles with ultra-small sizes below $5 \mathrm{~nm}$ were simply obtained in a similar manner (Fig. 2A-C). Extremely small silver nanoparticles are uncommon in the literature but when they occur [18] their synthesis is
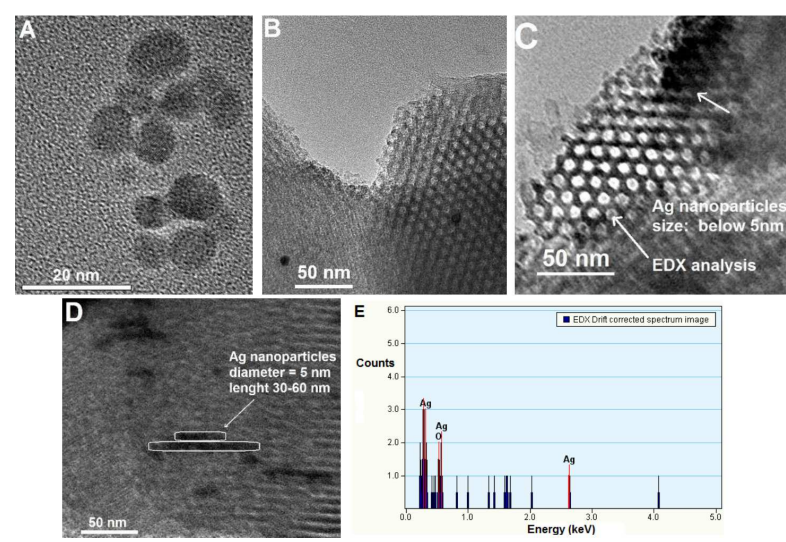

Fig. 2. (A,B) TEM images of the prepared silver nanoparticles on SBA-15, (C) Silver nanoparticles located on pores of silica, (D) silver nanoparticles located along mesopores of SBA-15, (E) EDX analysis from single silver nanoparticles from point marked on image (C).

often much more complicated than process proposed in this work.

Such small silver nanoparticles mean highly developed surface area, a larger fraction of the surface atoms, greater reactivity, and antimicrobial activity. In this case we observed that nanoparticles can be located both on the surface and inside pores of the support. When nanoparticles are deposited inside the pores, the size of voids determine the dimension and morphology of noble metal nanoparticles. Moreover, crystallization of long silver structures was observed when the amount of silver ions adsorbed on the support surface was at least three times greater than in the first case (\% content of $\mathrm{Ag}$ determined by XRF analysis $=4.56 \%$ ) (Fig. 2D). In both cases TEM images confirmed the ordered system of pores in mesoporous support which plays important role in the stabilization of the metallic nanostructures. Crystallites may be oriented inside the pores or along the mesopores of silica.

Palladium/silica composites contained well defined palladium crystallites with sizes up to $10 \mathrm{~nm}$ (Fig. 3A).

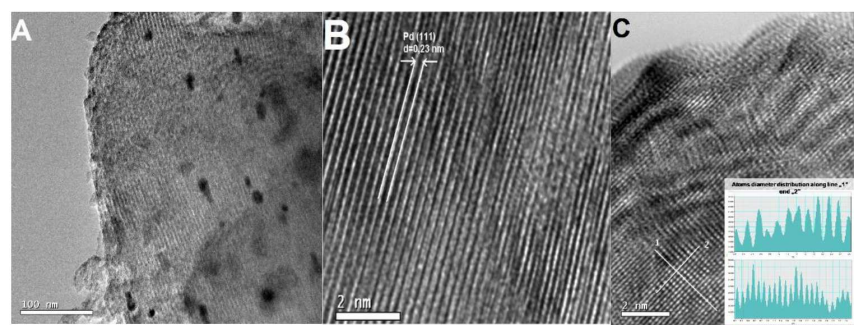

Fig. 3. (A) TEM images of palladium nanoparticles on SBA-15 surface, (B,C) HRTEM images of palladium nanoparticles with well-defined lattice planes.

In this instance, the formation of explicit polycrystalline form was observed instead of well separated, single 
nanoparticles. However, palladium nanoparticles are still very uniformly distributed over the surface of the carrier. HRTEM images (Fig. 3B,C) shows well defined lattice planes (first lattice planes $\mathrm{Pd}(111)$ with interplanar spacing $d_{P d(111)}=0.23 \mathrm{~nm}$ and distribution of the palladium atoms on these lattice planes (Fig. 3C).

When the second type of support was applied (mesoporous aluminosilicate) we observed a significant difference in the size and shape of final noble metal nanoparticles in comparison to SBA-15. The noble metal nanoparticles were still uniformly distributed on the support. Palladium nanoparticles (Fig. 4A,B) were significantly smaller (diameter of particles was about $5 \mathrm{~nm}$ ), while platinum nanoparticles were larger (diameter of platinum nanoparticles approximately $10 \mathrm{~nm}$ ). Some part of platinum nanoparticles were in the form of triangular nanoparticles. This shape was not observed in the case of SBA-15. High resolution TEM image of platinum nanoparticles (Fig. 4E) shows crystal lattice projections with distance suitable for $\mathrm{Pt}(111)$ lattice planes. Finally, noble metal nanoparticles remained in the form of welldefined.

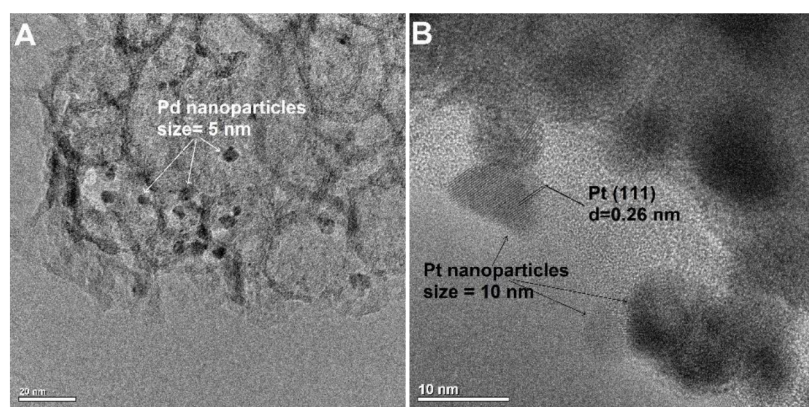

Fig. 4. (A) TEM images of palladium nanoparticles on mesoporous aluminosilicate, (B) HRTEM image of platinum nanoparticles.

\section{Conclusions}

Incorporation of the noble metal nanoparticles into the material, which is chemically inert with respect to other materials and harmless to humans causes growing its competitiveness and practical use in catalysis and medical applications. Moreover, the deposition of nanoparticles on the solid material increases their stability and makes them in state of high dispersion. By careful selection of the carrier material it is possible to obtain nanoparticles with various sizes and shapes. Production and further application seems to be easier if the selected precursor of metallic nanoparticles allows to obtain metallic nanoforms in a relatively simple, inexpensive and reproducible way.

\section{References}

[1] G. Sergeev, T. Shabatina, Colloids Surf. A Physicochem. Eng. Asp. 313, 18 (2008).

[2] S. Guo, E. Wang, Nano Today 6, 240 (2011).

[3] J.M. Nam, C.C. Thaxton, C.A. Mirkin, Science 301, 1884 (2003).

[4] O.V. Salata, J. Nanobiotechnol. 2, 3 (2004).

[5] W. Park, K. Na, Wiley Interdiscipl. Rev. Nanomed Nanobiotechnol. 7, 494 (2015).

[6] N. Piri, A. Shams-Nateri, J. Mokhtari, Color Res. Appl. (2015).

[7] Y. Sun, G.Z. Guo, Y. Liu, G. Zhao, L. Zhang, H. Jiu, Q. Zhang, Curr. Nanosci. 6, 103 (2010).

[8] S.M. El-Sheikh, A.A. Ismal, J. Al-Sharab, New J. Chem. 37, 2399 (2013).

[9] S. Schauermann, N. Nilius, S. Shaikhutdinov, H.-J. Freund, Acc. Chem. Res. 46, 1673 (2013).

[10] D. Astruc, F. Lu, J.R. Aranzaes, Andew. Chem. Int. Ed. 44, 7852 (2005).

[11] Z. Ma, S. Dai, Heterogen. Gold Catalysts Catal. 1 (2014).

[12] L.C. Gontard, A. Fernández, R.E. Dunin-Borkowski, T. Kasama, S. Lozano-Pérez, S. Lucas, Micron 6, 1 (2014).

[13] A.A. Herzing, A.F. Carley, J.K. Edwards, G.J. Hutchings, C.J. Kiely, Chem. Mater. 20, 1492 (2008).

[14] M. Zienkiewicz-Strzałka, S. Pikus, Appl. Surf. Sci. 261, 616 (2012).

[15] M. Zienkiewicz-Strzałka, S. Pikus, Appl. Surf. Sci. 265, 904 (2013).

[16] M. Zienkiewicz-Strzałka, S. Pasieczna-Patkowska, M. Kozak, S. Pikus, Appl. Surf. Sci. 266, 337 (2013).

[17] M. Zienkiewicz-Strzałka, A. Deryło-Marczewska, S. Pikus, Micropor. Mesopor. Mater. 277, 228 (2016).

[18] L. Du, Q. Xu, M. Huang, L. Xian, J.X. Feng, Mater. Chem. Phys. 160, 40 (2015). 